\title{
Combining Operando X-ray Absorption Spectroscopy and Sub-Ångstrom Environmental Electron Microscopy
}

\author{
Eric A. Stach
}

Center for Functional Nanomaterials, Brookhaven National Laboratory, Upton, NY 11733

Understanding the atomic-scale response of materials in reactive environments is crucial to advancing several of the world's most pressing energy needs, specifically in the areas of catalysis, energy conversion and energy storage. Here, we describe a proposed project at the National Synchrotron Light Source II to create a combination of operando X-ray absorption spectroscopy (XAS) and in situ / environmental transmission electron microscopy (ETEM) in the same instrument (XEM). This system would allow sub-Angstrom correlation between the dynamics of atomic and electronic structure changes that occur in materials in response to complex, reactive environments. By allowing an x-ray beam to pass through the microscope's objective pole-piece, we would be able to acquire simultaneously - high-brightness $\mathrm{x}$ ray spectroscopic information and integrate this directly with highresolution electron-based characterization at the atomic scale. The resulting information will provide unprecedented insights into the atomistic and electronic structure of individual active sites involved in chemical reactions, and "Grand Challenge" in energy research.

By examining the near-edge structure (within a several tens of eV) from the edge onset (XANES) one can understand how the electronic density of states in a material changes during chemical reactions. Additionally, investigation of the energy loss spectrum after this near edge region can be used to interrogate the local atomic environment of the sample. Importantly these approaches allow interrogation of electronic structure in reactive environments. By combining XANES and EXAFS acquired in the same measurement - one can obtain independent complementary knowledge of particle size, details of the electronic states and band character of catalytic materials. This information is considered crucial to understanding both catalyst performance and catalyst deactivation, and as such is a primary technique in the field.

Despite the power and wide-spread application of this approach, the interpretation of XAS spectra often requires specific assumptions to be made concerning the morphology of the nanoparticles, including size, shape and composition. Crucially, these models often make assumptions regarding the heterogeneity of both size and shape during reactions. Yet it is rare that these assumptions are accompanied by independent experimental verification, as there is no available technique that allows high-resolution images of nanoparticles in their reactive environments at the same time.

This is the crux of the proposed instrumentation: by combining these approaches we will be able to imaging concomitantly dynamic changes in morphology (size, shape and composition) in support nanoparticle systems during chemical reactions and correlate these observations with the changes in the electronic structure that mediate these reactions. This experimental data - when combined with appropriate atomistic simulations - will provide the key insights into how catalysis works at the atomic scale, providing crucial information needed to design new materials systems for improved performance. This presentation will motivate the instrument - specifically the need for combined characterization will describe its configuration and anticipate experiments that it could perform. 
[1] The author would like to that colleagues who have helped in the development of these ideas. They include: Simon Bare, Philip Batson, Steven Bradley, Nigel Browning, Jingguang Chen, Abhaya Datye, Anatoly Frenkel, Bernd Kabius, Christopher Marshall, Jeffery Miller, Fabio Riberio, José Rodriguez, Miquel Salmeron, Judith Yang and Yimei Zhu. Critical insights during the review process from David Muller are also acknowledged.

[2] Research carried out at the Center for Functional Nanomaterials, Brookhaven National Laboratory, which is supported by the U.S. Department of Energy, Office of Basic Energy Sciences, under Contract No. DE-AC02-98CH10886

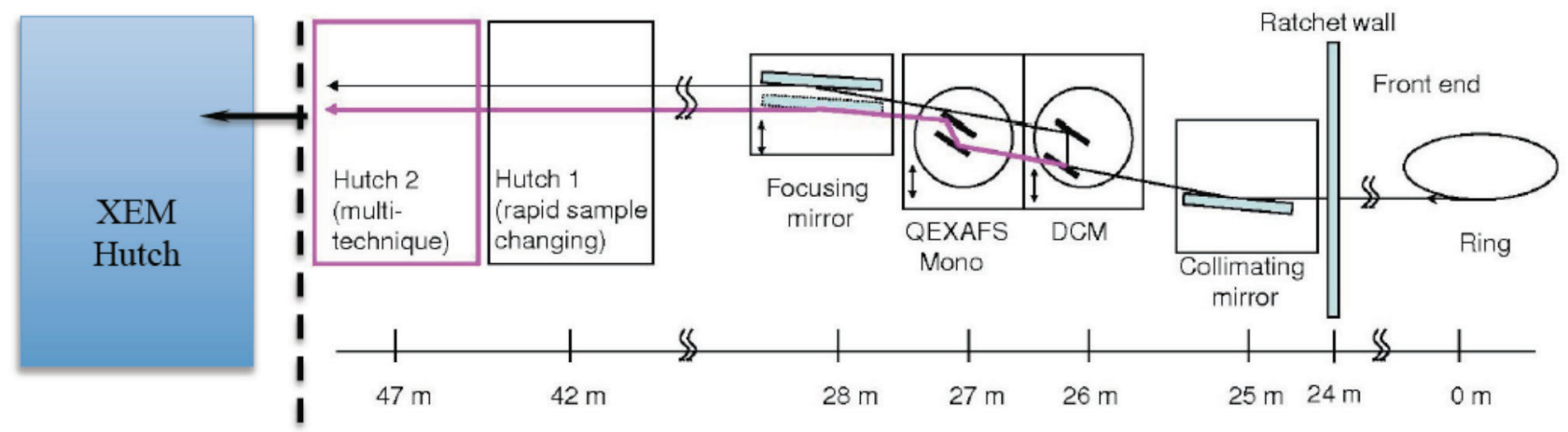

Figure 1. Schematic of integration of XEM with proposed design for the "Quick X-ray Absorption and Scattering Beamline at the NSLS-II (QAS). The beamline source for QAS will be a 3-pole wiggler and XEM build upon the existing beamline optics, which are slated to include a collimating mirror (allowing energy rangs from 4.5 to $34 \mathrm{kV}$ ), a double-crystal, channel cut monochromator for in-situ quick EXAFS, and a toroidal focusing mirror.
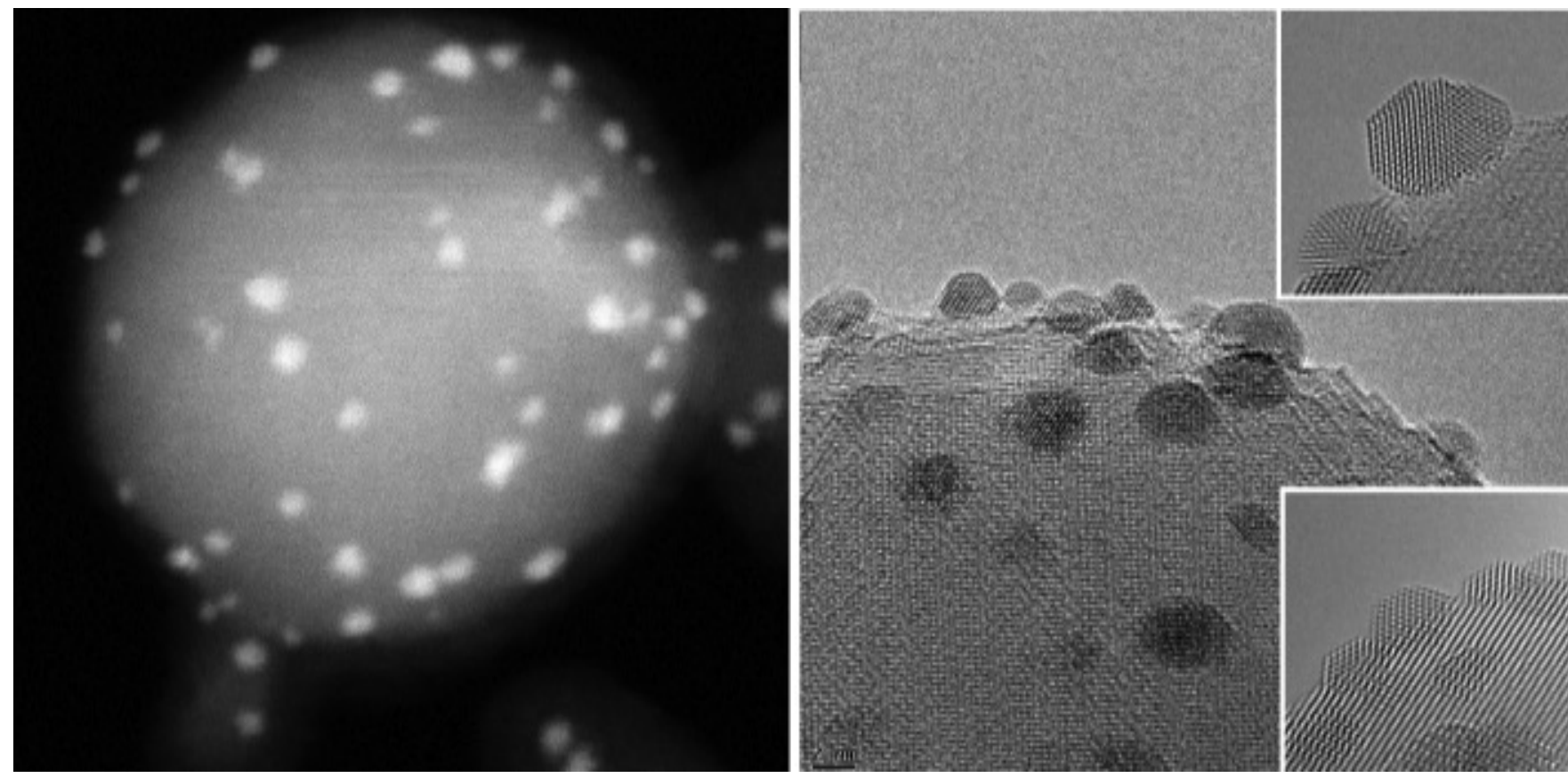

Figure 2. Critical to the correlative XAS / TEM approach is the creation of appropriate sample geometries. Here, we show images from our round, non-porous supports (in this case $\mathrm{Al}_{\mathrm{x}} \mathrm{O}_{\mathrm{y}}$, though we have made many others). This geometry leads to situations where there are always particles in projection, and which have sufficient density for XAS characterization. 